\title{
Anatomical variations of the innervated radial artery superficial palmar branch flap: A series of 28 clinical cases
}

\author{
Jae-Won Yang \\ Gangnam Jaejun Plastic Clinic for Hand \& Microsurgery, Pyeongtaek, Korea
}

Background The innervated radial artery superficial palmar branch (iRASP) flap was designed to provide consistent innervation by the palmar cutaneous branch of the median nerve (PCMN) to a glabrous skin flap. The iRASP flap is used to achieve coverage of diverse volar defects of digits. However, unexpected anatomical variations can affect flap survival and outcomes.

Methods Cases in which patients received iRASP flaps since April 1, 2014 were retrospectively investigated by reviewing the operation notes and intraoperative photographs. The injury type, flap dimensions, arterial and neural anatomy, secondary procedures, and complications were evaluated.

Results Twenty-eight cases were reviewed, and no flap failures were observed. The observed anatomical variations were the absence of a direct skin perforator, large-diameter radial artery superficial palmar branch (RASP), and the PCMN not being a single branch. Debulking procedures were performed in 16 cases (57.1\%) due to flap bulkiness.

Conclusions In some cases, an excessively large RASP artery was observed, even when there was no direct skin perforator from the RASP or variation in the PCMN. These findings should facilitate application of the iRASP flap, as well as any surgical procedures that involve potential damage to the PCMN in the inter-thenar crease region. Additional clinical cases will provide further clarification regarding potential anatomical variations.

Keywords Radial artery superficial palmar branch / Palmar cutaneous branch / Median Nerve / Finger injury / Perforator flap
Correspondence: Jae-Won Yang Gangnam Jaejun Plastic Clinic for Hand \& Microsurgery, 38 Pyeongtaek 5-ro 20beon-gil, Pyeongtaek 17902, Korea

Tel: +82-31-6628-3119

Fax: +82-31-6628-3199

E-mail: jwonyang@gmail.com

\section{INTRODUCTION}

The innervated radial artery superficial palmar branch (iRASP) flap was introduced in 2010. An iRASP flap provides a large area of glabrous skin and consistent innervation of the palmar cutaneous branch of the median nerve (PCMN), which is supplied by the direct skin perforators of the radial artery superficial pal- mar branch (RASP). The flap is used to cover volar defects of the digits [1].

The iRASP flap was formerly described as the RASP flap. Its anatomical characteristics, determined by a latex-injection study involving 30 fresh frozen cadavers, are as follows: the donor artery of the RASP is of similar size to a typical digital artery, the RASP has direct skin perforators that supply the PCMN and 
skin flap, and the PCMN is a single branch from the median nerve (Fig. 1A).

Unusual anatomical variations in the RASP and the PCMN are encountered. We investigated anatomical variations in the artery and nerve with the aim of enhancing the accuracy and safety of dissection.

\section{METHODS}

The indication for an iRASP flap was a volar defect in a digit of $>1.5 \mathrm{~cm}$ in width and $3.5 \mathrm{~cm}$ in length, with or without a pulp tissue defect. Patients with longstanding underlying diseases, such as hypertension, diabetes mellitus, or autoimmune diseases, were excluded.

The scaphoid tubercles were digitally palpated before surgery. Throbbing of the direct skin perforator was checked. To identify the origin of the RASP, ultrasonography was performed on the area between the radial artery and inlet site between the thenar muscles, even though the fine direct skin perforators were not visualized.

The most important surface landmarks when designing the iRASP flap are the scaphoid tubercle, radial side of the ring finger, contours of the flexor carpi radialis and palmaris longus tendons, and the radial styloid process. An elliptical flap is easily placed along a longitudinal axis from the radial side of the ring finger to the scaphoid tubercle. The radial side of the scaphoid tubercle, and $0.5 \mathrm{~cm}$ of its ulnar side from the interthenar crease, must be included in the width of the flap to ensure that no injury is sustained by the direct skin perforators over the scaphoid tubercle or by the PCMN along the interthenar crease. The length of the flap was adjusted to match that of the defect (Fig. 1B). The flap was dissected, starting from its radial side, to identify the RASP. Any subcutaneous veins encountered should be preserved in cases of weak concomitant veins. The PCMN was isolated between the flexor carpi radialis and palmaris longus tendons. After preparing the donor artery, nerves, and subcutaneous veins, the skin flap was elevated from the distal part above the palmar aponeurosis. The region of the scaphoid tubercle should be focused on because the direct skin perforators are densely attached to the fascia of the thenar muscle and roof of the flexor carpi radialis tendon. Further dissection of the arterial pedicle was carried out to the origin of the RASP. The flap was detached after ligating the arteries and concomitant veins (Supplemental Video 1).

Clinical cases after April 1, 2014 were reviewed by evaluating the operation notes and intraoperative photographs. Written informed consent was obtained from all patients. The mechanism of trauma, flap dimensions, and patterns of the RASP and PCMN were assessed, as were postoperative complications and secondary operations.

\section{RESULTS}

In total, 28 cases were included ( 22 male and 6 female patients),

\section{Fig. 1. PCMN type I and flap design}

(A) Type I variation in the palmar cutaneous branch of the median nerve (PCMN). Type I has a single branch of the PCMN between the flexor carpi radialis (FCR) and palmaris longus (PL) tendons, beneath the antebrachial fascia. A single branch is shown longitudinally attached to the undersurface of the elevated flap. (B) Design of the innervated radial artery superficial palmar branch (iRASP) flap. The most important surface landmarks are the scaphoid tubercle, radial side of the ring finger, contours of the FCR and PL tendons, and radial styloid process. An elliptical flap is easily placed along a longitudinal axis from the radial side of the ring finger to the scaphoid tubercle. The radial side of the scaphoid tubercle, and $0.5 \mathrm{~cm}$ of its ulnar side from the interthenar crease, must be included in the width of the flap to ensure that no injury is sustained by the direct skin perforators over the scaphoid tubercle or by the PCMN along the interthenar crease. The length of the flap can be adjusted to the length of the defect.
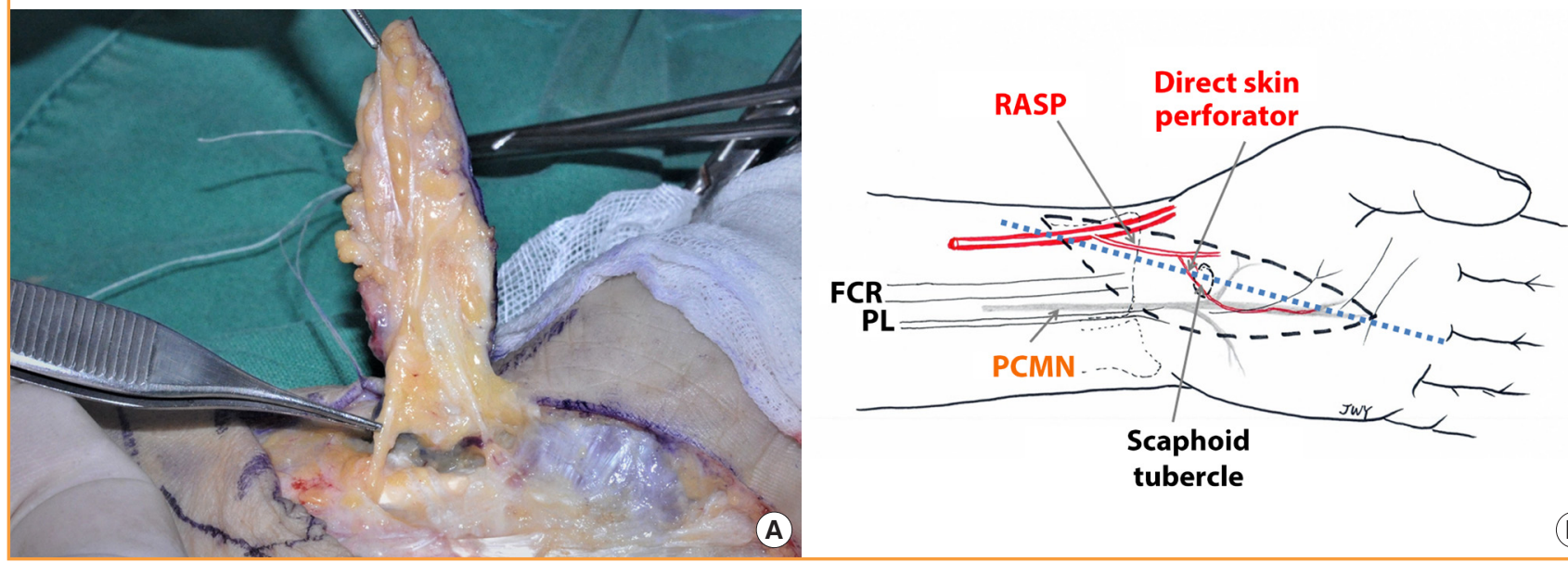
with a mean age of 46.6 years. The left hand was involved in 13 cases and the right hand was involved in 15 cases; the total number of injured digits was 28 . The most commonly injured digit was the index finger ( 12 cases, $42.8 \%$ ), followed by the long finger (seven cases, $25 \%$ ), thumb (five cases, $17.8 \%$ ), and small finger (three cases, 10.7\%). Only one case (3.5\%) involved a palm defect (Table 1). The mechanisms of injury were crushing (10 cases, $35.7 \%$ ), rotating blade (nine cases, $32.1 \%$ ), avulsion (seven cases, $25 \%$ ), friction (one case, $3.5 \%$ ), and burn (one case, 3.5\%) (Table 2). The mean width and length of the flaps were $2.09 \pm 0.19 \mathrm{~cm}$ and $6.55 \pm 1.48 \mathrm{~cm}$, respectively. The greatest width was $2.5 \mathrm{~cm}$ and the greatest length was $11 \mathrm{~cm}$. Among

\section{Table 1. Distribution of injured digits $(n=28)$}

\begin{tabular}{|lc|}
\hline Variable & No. of cases $(\%)$ \\
\hline Thumb & $5(17.8)$ \\
Index & $12(42.8)$ \\
Long & $7(25.0)$ \\
Ring & 0 \\
Small & $3(10.7)$ \\
Palm & $1(3.5)$ \\
\hline
\end{tabular}

\section{Table 2. Mechanisms of injury $(n=28)$}

\begin{tabular}{|lc|}
\hline Variable & No. of cases $(\%)$ \\
\hline Crushing & $10(35.7)$ \\
Rotating blade & $9(32.1)$ \\
Avulsion & $7(25.0)$ \\
Friction & $1(3.5)$ \\
Burn & $1(3.5)$ \\
\hline
\end{tabular}

the volar finger defects, pulp lesions were covered in 17 cases (60.7\%). A flow-through anastomosis was performed in two cases. In one case, an interpolation flap was used without microanastomosis. In 15 cases (53.6\%), coverage was achieved by an emergency flap, which was defined as an operation performed within 48 hours after trauma.

The origin of the RASP from the radial artery was, on average, $1.30 \pm 0.59 \mathrm{~cm}$ proximal from the radial styloid process. The closest origin was $0.2 \mathrm{~cm}$ and the farthest origin was $2.2 \mathrm{~cm}$ (Fig. 2). The mean external diameter was $1.02 \pm 0.35 \mathrm{~mm}$. The smallest diameter was $0.6 \mathrm{~mm}$ and the largest diameter was $2.0 \mathrm{~mm}$ (Figs. 3, 4). Only one case (3.6\%) showed no direct skin perforator from the RASP. Three cases exhibited an excessive diameter (> $1.5 \mathrm{~mm}$; mean $1.8 \pm 0.14 \mathrm{~mm})(10.7 \%)$.

Based on the numbers of PCMN branches and mixed innervation with other nerves, the cases were divided into types I, II, and III (Table 3). Type I comprised PCMNs with a single branch and was observed in 17 cases (60.7\%) (Fig. 1). The remaining 10 cases were type II, in which two distinctive branches separately coursed along the superficial and deep planes. The superficial branch was smaller than the deep branch (type IIa) in five cases. The superficial and deep branches were of similar size (type IIb) in five cases. Only 1 case (3.6\%) was type III and exhibited mixed innervation with a PCMN and lateral antebrachial cutaneous (LABC) nerve of similar diameter.

All flaps survived. In one case, a hematoma formed below the flap and in another case, sustained hematoma was observed at the donor site. Debulking procedures were performed in 16 cases $(57.1 \%)$. There were no cases with a significant neuroma at

\section{Fig. 2. Origin site of the RASP}

The radial artery superficial palmar branch (RASP) originated from the radial artery at a mean of $1.30 \pm 0.59 \mathrm{~cm}$ proximal to the radial styloid process. The closest origin was $0.2 \mathrm{~cm}$ and the farthest origin was $2.2 \mathrm{~cm}$. Two common sites are shown at 0.5 and $1.2 \mathrm{~cm}$ proximal to the radial styloid process.

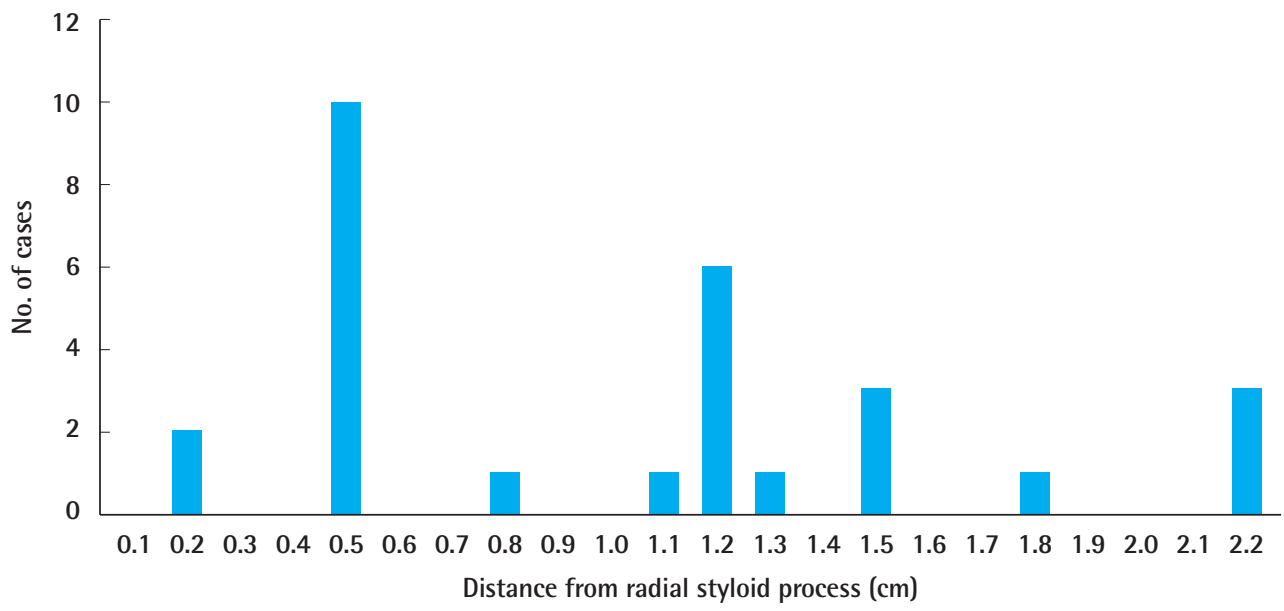




\section{Fig. 3. External diameter of the RASP}

The mean external diameter was $1.02 \pm 0.35 \mathrm{~mm}$; the smallest diameter was $0.6 \mathrm{~mm}$ and the largest diameter was $2.0 \mathrm{~mm}$. RASP, radial artery superficial palmar branch.

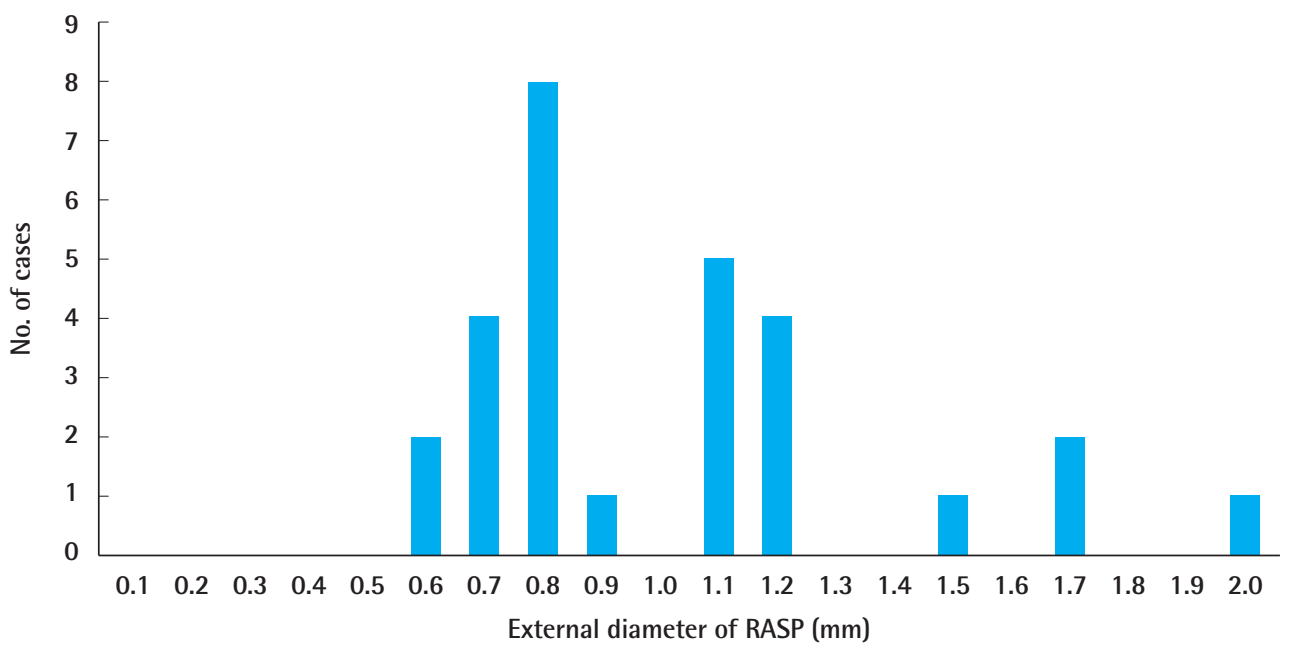

\section{Fig. 4. The largest RASP}

The largest radial artery superficial palmar branch (RASP) was 2.0 $\mathrm{mm}$ in diameter (yellow ribbon) with prominent concomitant veins. The radial artery and its concomitant veins (white arrow) are shown behind the RASP.

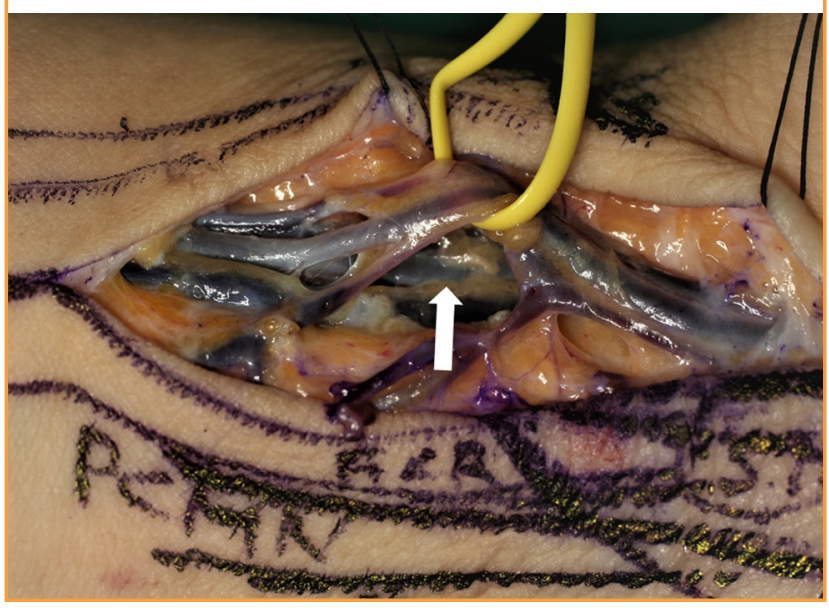

the cut end of the PCMN. Tightness in the wrist was observed during the early postoperative period, but subsided spontaneously. Other complaints comprised hypertrophic scars at the donor site. Conservative management with triamcinolone injection reduced the scarring and discomfort.

\section{Cases involving anatomical variations}

\section{Case 1. No direct skin perforator from the RASP}

One case had no direct skin perforator from the RASP. Fortunately, the arteries hitch-hiking with the superficial and deep branches of the PCMN could be anastomosed with the ulnar

\section{Table 3. Variations of PCMN innervation $(n=28)$}

\begin{tabular}{|c|c|c|}
\hline Type & Characteristics of innervation & No. $(\%)$ \\
\hline I & Single superficial branch of the PCMN & $17(60.7)$ \\
\hline \multicolumn{3}{|l|}{$\|$} \\
\hline Ila & Thin superficial|al) and thick deep branch of the PCMN & $5(17.9)$ \\
\hline$\| \mathrm{llb}$ & $\begin{array}{l}\text { Superficial and deep branches of the PCMN with similar } \\
\text { thickness }\end{array}$ & $5(17.9)$ \\
\hline |II & Mixed innervation with the LABC & $1(3.6)$ \\
\hline
\end{tabular}

The cases were divided into types $1-I I I$ according to the number of PCMN branches and the pattern of mixed innervation with other nerves.

PCMN, palmar cutaneous branch of the median nerve; LABC, lateral antebrachial cutaneous.

a)The superficial branch of the PCMN penetrated the superficial transverse carpal ligament just proximally to the wrist crease and coursed into the flap superficially; ${ }^{b}$ The deep branch of the PCMN coursed beneath the superficial transverse carpal ligament, crossed the wrist crease, and penetrated the palmar aponeurosis; it innervated distal skin further from the wrist crease compared to the thin superficial branch.

proper digital artery. The artery accompanying the deep branch of the PCMN was estimated to have an external diameter of 0.4 mm (Fig. 5).

\section{Case 2. RASP of excessive diameter}

The external diameter was $2.0 \mathrm{~mm}$ in this case, which hindered anastomosis. This limitation was addressed by using a horizontal mattress suture technique with an unequal bite width after an oblique cut of the smaller recipient artery (Fig. 6, Supplemental Video 2).

\section{Case 3. Type II variation in the PCMN}

This case was type IIa and involved a thin superficial and a thick 


\section{Fig. 5. No direct skin perforators from the RASP}

(A) A 51-year-old man sustained a large volar skin and soft tissue defect on the left index finger by a milling machine. An innervated radial artery superficial palmar branch (iRASP) flap was designed for emergency coverage of the defect. (B) However, there was no direct skin perforator from the RASP supplying the elevated flap. Therefore, the elevated flap was separated from the pedicle of the RASP. This case involved a type Ila variation in the palmar cutaneous branch of the median nerve (PCMN) (a thin superficial and a thick deep branch) on the undersurface of the elevated flap. After careful dissection of the branches of the PCMN, the accompanying arteries (white arrow) were identified. (C) Anatomical structures. No connection was observed between the RASP and PCMN (red dotted region). The accompanying artery of the thick deep branch of the PCMN was anastomosed with the recipient ulnar proper digital artery. (D) The covered donor site at 2 months after surgery. PL, palmaris longus.
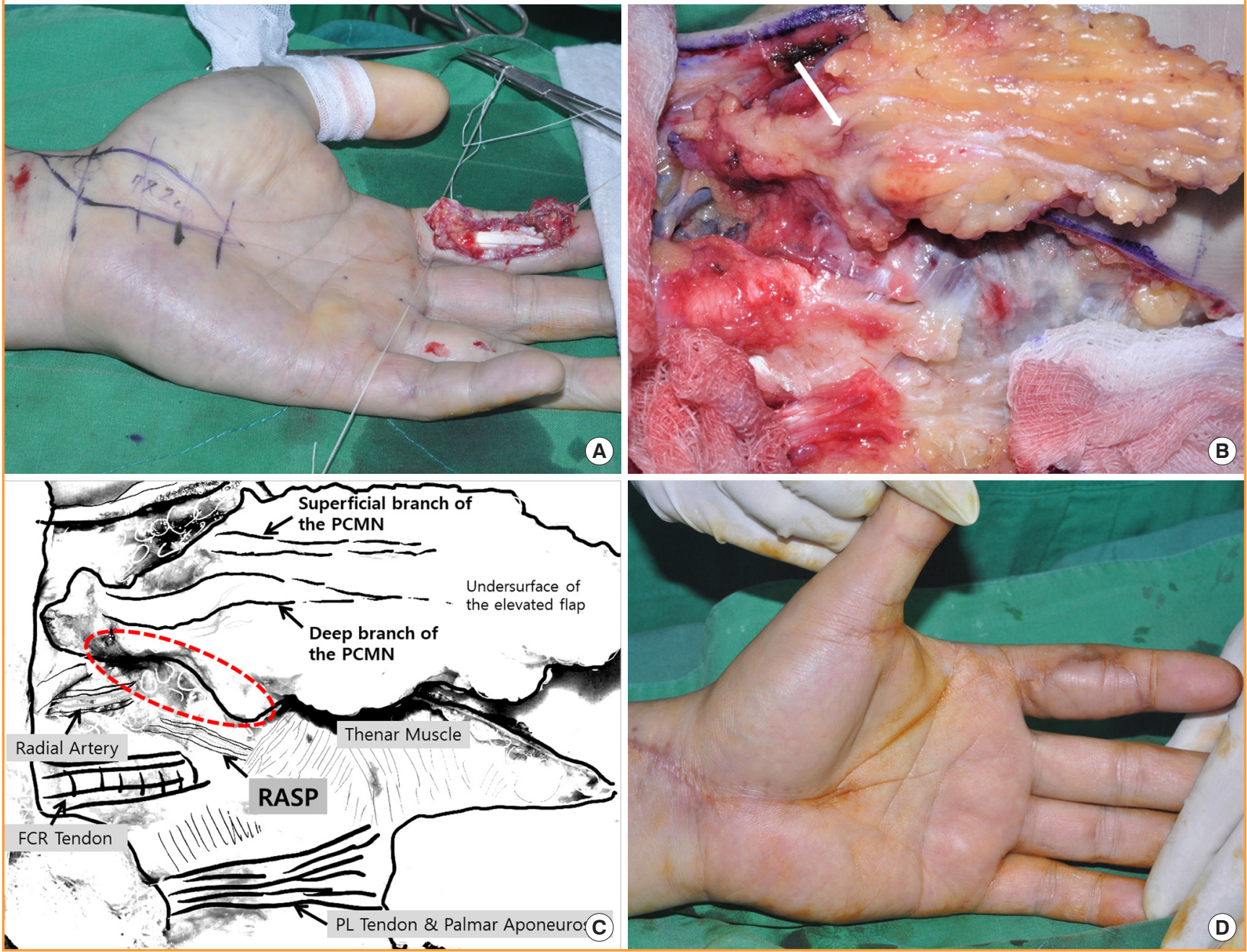

Fig. 6. RASP of excessive diameter

(A) The external diameter of the radial artery superficial palmar branch (RASP) was estimated to be $2.0 \mathrm{~mm}$. (B) The size discrepancy was resolved by using a horizontal mattress suture after an oblique cut of the smaller proper digital artery.
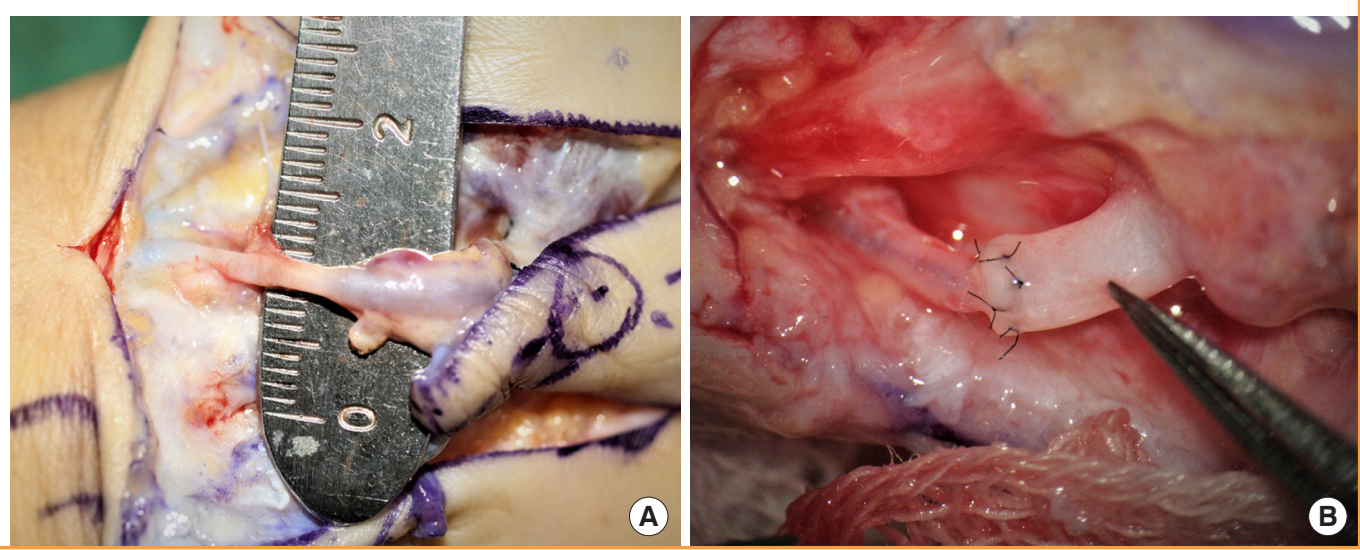


\section{Fig 7. Type Ila variation in the PCMN}

(A) This case was type lla: a thin superficial (thin arrow) and thick deep branch (thick arrows) of the palmar cutaneous branch of the median nerve (PCMN). (B) Courses of the superficial and deep branches of the PCMN. The superficial branch of the PCMN penetrated the superficial transverse carpal ligament proximal to the wrist crease and coursed into the flap dimension. The deep branch of the PCMN coursed beneath the superficial transverse carpal ligament, crossed the wrist crease, and penetrated the palmar aponeurosis; it innervated distal skin further from the wrist crease compared to the thin superficial branch. PL, palmaris longus.

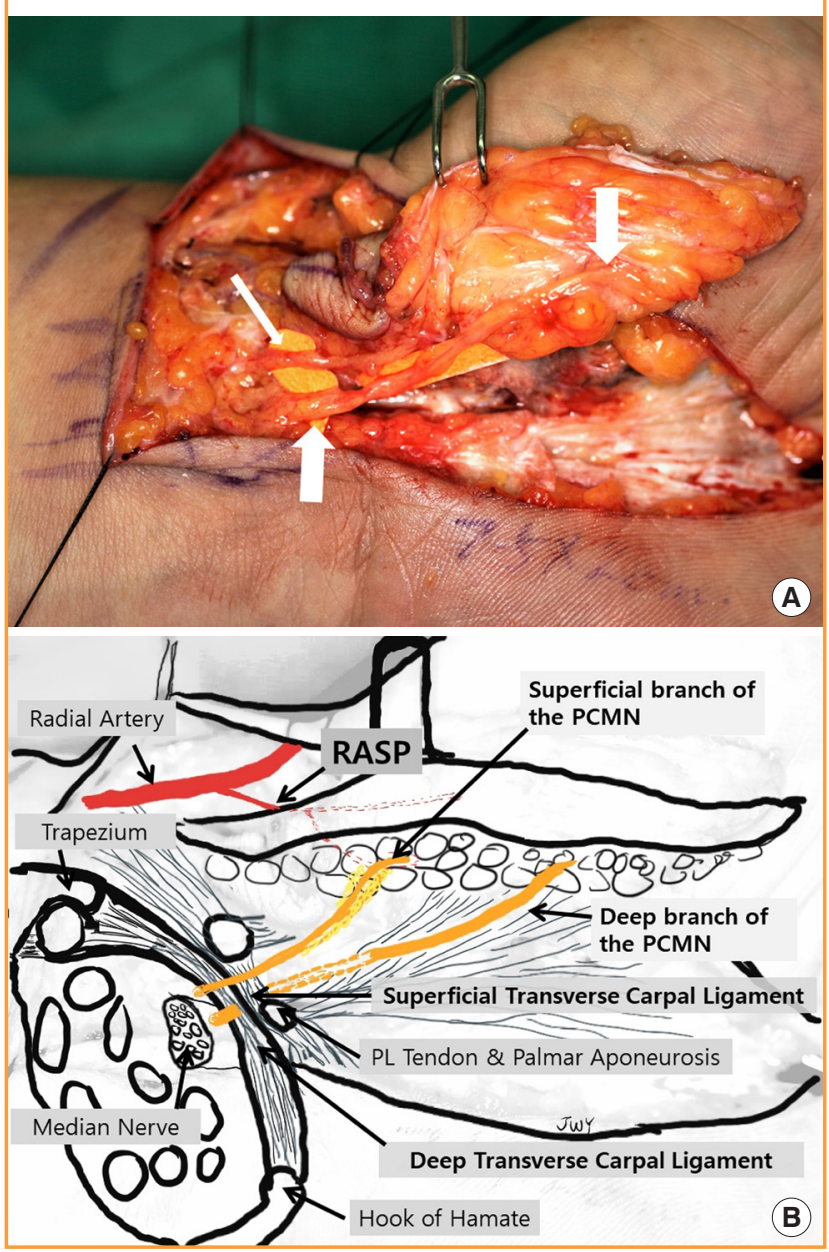

deep branch of the PCMN. The superficial branch of the PCMN passed through the superficial transverse carpal ligament immediately proximal to the crease of the wrist and coursed into the flap superficially. The deep branch of the PCMN coursed beneath the superficial transverse carpal ligament, crossed the crease of the wrist, and penetrated the palmar aponeurosis; it innervated distal skin further from the wrist crease compared to the thin superficial branch (Fig. 7).

\section{Case 4. Type IIb variation in the PCMN}

This case involved two branches of the PCMN of similar size and was defined as a type IIb variation. The courses of the su-

\section{Fig. 8. Type Ilb variation in the PCMN}

This case involved two palmar cutaneous branch of the median nerve (PCMN) branches of a similar size. The superficial branch (thin arrow) coursed superficially and the deep branch (thick arrow) coursed deeply, similar to type lla.

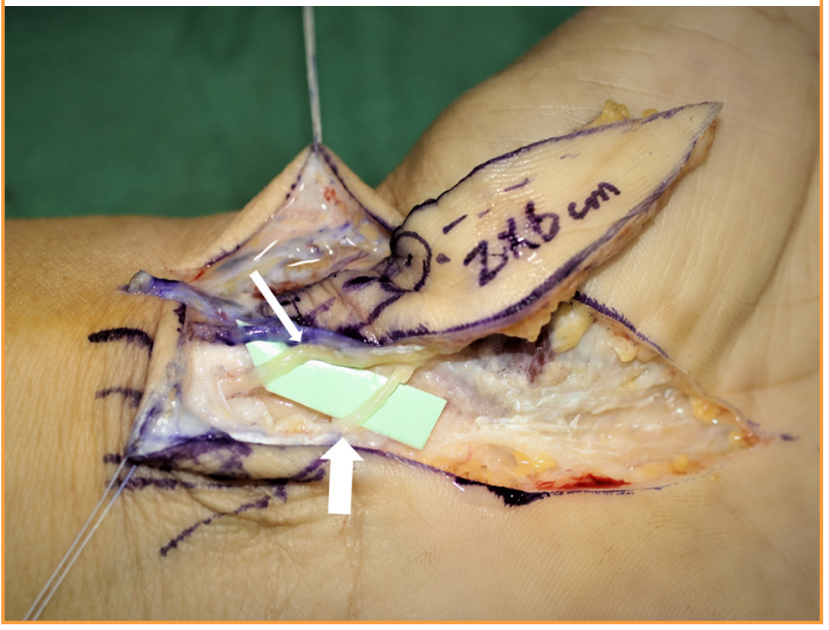

\section{Fig. 9. Type III variation in the PCMN}

This case involved a thick prominent branch of the lateral antebrachial cutaneous nerve (LABC) (thin arrow) that coursed with the radial artery in the distal forearm. It was located on the radial side of the flexor carpi radialis tendon. The $L A B C$ coursed to the ulnar side and innervated the elevated flap dimension. Type III variation was defined as mixed innervation with the palmar cutaneous branch of the median nerve (PCMN) (thick arrow) and LABC of similar diameter.

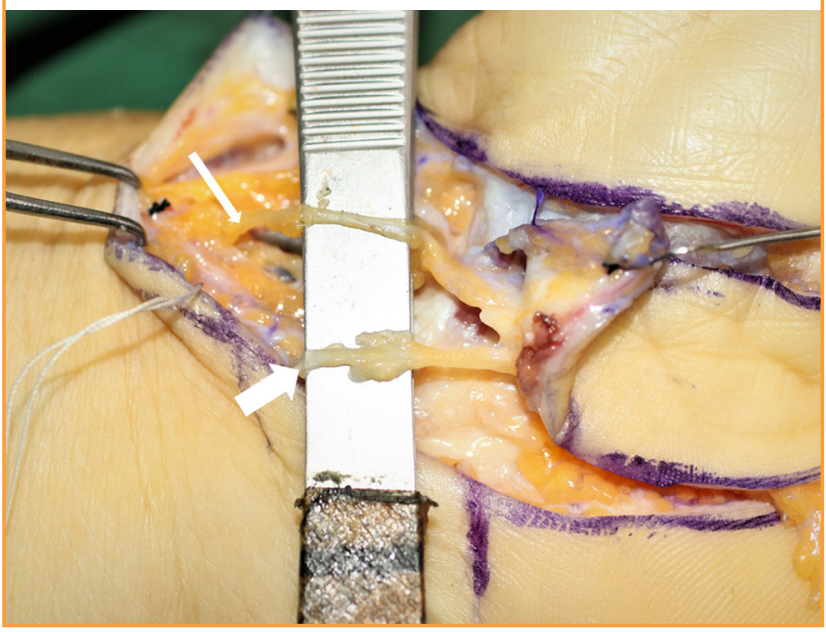

perficial and deep branches were identical to those in type IIa (Fig. 8).

\section{Case 5. Type III variation in the PCMN}

This case involved a prominent branch of the LABC coursing with the radial artery. Type III was defined as involving mixed innervation with a PCMN and LABC of similar diameter (Fig. 9). 


\section{DISCUSSION}

Historically, the RASP has been used in several flaps as a donor artery. The free thenar flap was first developed [2], followed by the innervated radial thenar flap [3] and the superficial palmar branch of the radial artery (SPBRA) flap [4]. The free thenar flap uses the LABC nerve for innervation, which is inconsistent and slender. The innervated radial thenar flap uses more radial skin to include the superficial sensory nerve of the radial nerve. The SPBRA flap uses a portion of the PCMN, because the SPBRA flap courses transversely along the wrist crease and a nonglabrous skin flap is used [5].

In terms of nomenclature, we recommend using the term "iRASP flap" to prevent confusion with the SPBRA flap. The SPBRA flap should be used only when the donor site is nonglabrous skin of the wrist and courses transversely. The lowercase "I" in the term "iRASP flap" is an abbreviation for "innervated," which implies better sensation with consistent innervation of the PCMN and its glabrous skin flap. This is why the "RASP flap" is now termed the "iRASP flap." Because the direct skin perforators from the RASP artery were identified in a previous cadaver study, the iRASP flap was defined as a perforator flap, while previous flaps were defined as fasciocutaneous flaps $[2,3]$. Hence, this flap was named "iRASP" based on the source artery of the SPBRA, in accordance with the Gent consensus [6]. To aid memory, the acronym "RASP" may remind surgeons of tools used to smooth the surface of bone.

The iRASP flap is popular in South Korea $[7,8]$. In one case report, the iRASP flap was used in combination with the SPBRA flap to cover a totally degloved finger [9]. The unique characteristics of the iRASP flap (glabrous skin, consistent innervation of the PCMN, and a close anatomical relationship between direct skin perforators and the PCMN) make it attractive to hand surgeons.

The iRASP flap is affected by variations in the arterial anatomy at the higher forearm level and the superficial palmar arch of the hand. The median artery and superficial palmar arch of the hand exhibit high frequencies of variation $[10,11]$. We encountered a case with no direct skin perforator from the RASP to the PCMN. There are several types of superficial palmar arches $[12,13]$. The median artery can be identified in $23 \%$ of cases [14]. The median artery forms a superficial palmar arch with the ulnar artery; alternatively, it irrigates the thumb and index finger separately without forming a superficial palmar arch. In this situation, the median artery serves a vital function in supplying nutrients to the PCMN. The RASP does not contribute to the PCMN and therefore cannot hitch-hike with the PCMN. These findings are important for flap survival; thus, preopera- tive ultrasonography or vascular imaging is recommended.

The size of RASP at the origin from the radial artery was $1.02 \pm 0.35 \mathrm{~mm}$ in the present series, which is smaller than has been reported previously $(1.49 \pm 0.46 \mathrm{~mm})[1,11]$. This difference may have been due to shrinkage of the artery as a result of tourniquet use in the present series, or to expansion of the artery by the forceful injection of latex in the prior anatomical study. In that study, the hands and forearms of 30 adult fresh frozen cadavers were dissected under $\times 3.5$ loupe magnification approximately 12 hours after injecting a silicone rubber compound (Microfil MV-130; Flow Tech, Inc., Carver, MA, USA) through either the brachial artery or radial artery to enhance arterial anatomy. An excessively large RASP was also encountered in the present series (mean, $1.8 \pm 0.14 \mathrm{~mm}$ ), which hindered anastomosis. When the radial artery is the dominant artery that supplies the superficial palmar arch or the thumb and radial side fingers without the superficial palmar arch, the RASP is presumably larger than commonly observed.

The PCMN is problematic because the inter-thenar region is a common incision site and injury of the PCMN can result in considerable discomfort [15]. The PCMN has been described as a single branch originating from the radial side of the median nerve under the antebrachial fascia at the level of the distal forearm [16-20]. It courses superficially through the superficial transverse carpal ligament and innervates the palmar skin longitudinally along the inter-thenar crease. However, the PCMN was not a single branch in $35.7 \%$ of the cases in the present series. In addition, the inter-thenar region of the iRASP flap exhibited mixed innervation with the PCMN and LABC. Only one of the 28 cases involved a prominent, thick LABC. Increased attention is needed during nerve dissection, such as open and endoscopic carpal tunnel release [21,22], Camitz opponensplasty, or elevation of an iRASP flap. A neglected injury to the deep branch of the PCMN or the LABC is likely to cause discomfort. In terms of innervation of the iRASP flap, branches should be attached to improve sensory outcomes.

Further anatomical study is required to verify the arterial and neural anatomy, because this study had some limitations. The first limitation was that the results were confined to clinical cases from the Korean population. The second limitation was that dissection of pedicles must be confined to the operative field of the donor site.

In terms of anatomical variations of the iRASP flap, we observed an excessively large RASP artery, even when there was no direct skin perforator from the RASP or variation in the PCMN. These findings should facilitate application of the iRASP flap, as well as any surgical procedures that involve potential damage to the PCMN in the inter-thenar crease region. 
Additional clinical cases should confirm anatomical variation in the arteries and nerves.

\section{NOTES}

\section{Conflict of interest}

No potential conflict of interest relevant to this article was reported.

\section{Ethical approval}

This study was approved by the Institutional Review Board of the Korea National Institute for Bioethics Policy (IRB No. P01202003-21-001) and was performed in accordance with the principles of the Declaration of Helsinki. Written informed consents were obtained.

\section{Patient consent}

The patients provided written informed consent for the publication and the use of their images.

\section{ORCID}

Jae-Won Yang

https://orcid.org/0000-0003-1371-3440

\section{Supplementary material}

Supplemental Video 1. Design and dissection of the iRASP flap. Flap dissection usually started from the radial side of the flap, to identify the RASP. Any subcutaneous veins encountered should be preserved in cases of weak concomitant veins. The next step was to isolate the PCMN between the flexor carpi radialis and palmaris longus tendons. After preparing the donor artery, nerve and subcutaneous veins, the skin flap was elevated from the distal part above the palmar aponeurosis. The region of the scaphoid tubercle was the main focus, because the direct skin perforators were densely attached to the fascia of the thenar muscle and roof of the flexor carpi radialis tendon. Further dissection of the arterial pedicle was carried out to the origin of the RASP. The flap was detached after ligating the arteries and concomitant veins. PCMN, palmar cutaneous branch of the median nerve; iRASP, innervated radial artery superficial palmar branch; RASP, radial artery superficial palmar branch. Supplemental data can be found at: https://doi.org/10.5999/aps.2020.00423.v001.

Supplemental Video 2. Horizontal mattress anastomosis to resolve a size discrepancy. This technique ensures intima-to-intima contact for reliable patency. The horizontal mattress suture technique with unequal bite width can reduce the perimeter of a large-diameter vessel. The oblique cut of the small recipient artery increases the perimeter of the small vessel. Supplemental data can be found at: https://doi.org/10.5999/aps.2020.00423. v002.

\section{REFERENCES}

1. Yang JW, Kim JS, Lee DC, et al. The radial artery superficial palmar branch flap: a modified free thenar flap with constant innervation.J Reconstr Microsurg 2010;26:529-38.

2. Kamei K, Ide Y, Kimura T. A new free thenar flap. Plast Reconstr Surg 1993;92:1380-4.

3. Omokawa S, Mizumoto S, Iwai $M$, et al. Innervated radial thenar flap for sensory reconstruction of fingers. J Hand Surg Am 1996;21:373-80.

4. Sakai S. Free flap from the flexor aspect of the wrist for resurfacing defects of the hand and fingers. Plast Reconstr Surg 2003;111:1412-20.

5. Chi Z, Pafitanis G, Pont LEP, et al. The use of innervated radial artery superficial palmar branch perforator free flap for complex digital injuries reconstruction. J Plast Surg Hand Surg 2018;52:111-6.

6. Blondeel PN, Van Landuyt KH, Monstrey SJ, et al. The "Gent" consensus on perforator flap terminology: preliminary definitions. Plast Reconstr Surg 2003;112:1378-83.

7. Kim YJ, Suh YS, Lee SH, et al. The radial artery superficial palmar (RASP) branch free flap for finger soft tissue reconstruction. J Korean Soc Micorsurg 2012;21:21-6.

8. Lee SH, Cheon SJ, Kim YJ. Clinical application of a free radial artery superficial palmar branch flap for soft-tissue reconstruction of digital injuries. J Hand Surg Eur Vol 2017; 42:151-6.

9. Chi Z, Yang P, Song D, et al. Reconstruction of totally degloved fingers: a novel application of the bilobed spiraled innervated radial artery superficial palmar branch perforator flap design provides for primary donor-site closure. Surg Radiol Anat 2017;39:547-57.

10. Buch C, Devora CM, Johnson LY, et al. Incomplete superficial palmar arch and bilateral persistent median artery. Int J Surg Case Rep 2019;58:205-7.

11. Radunovic M, Vukcevic B, Radojevic N, et al. Superficial palmar branch of the radial artery: a study of occurrence and diameter. Ann Anat 2018;220:55-9.

12. Coleman SS, Anson BJ. Arterial patterns in the hand based upon a study of 650 specimens. Surg Gynecol Obstet 1961; 113:409-24.

13. Joshi SB, Vatsalaswamy P, Bahetee BH. Variation in formation of superficial palmar arches with clinical implications. J Clin Diagn Res 2014;8:AC06-9.

14. Olave E, Prates JC, Gabrielli C, et al. Median artery and su- 
perficial palmar branch of the radial artery in the carpal tunnel. Scand J Plast Reconstr Surg Hand Surg 1997;31:13-6.

15. Carroll RE, Green DP. The significance of the palmar cutaneous nerve at the wrist. Clin Orthop Relat Res 1972;83: 24-8.

16. Taleisnik J. The palmar cutaneous branch of the median nerve and the approach to the carpal tunnel. An anatomical study.J Bone Joint Surg Am 1973;55:1212-7.

17. Bezerra AJ, Carvalho VC, Nucci A. An anatomical study of the palmar cutaneous branch of the median nerve. Surg Radiol Anat 1986;8:183-8.

18. Hobbs RA, Magnussen PA, Tonkin MA. Palmar cutaneous branch of the median nerve. J Hand Surg Am 1990;15:3843.
19. DaSilva MF, Moore DC, Weiss AP, et al. Anatomy of the palmar cutaneous branch of the median nerve: clinical significance. J Hand Surg Am 1996;21:639-43.

20. Smith JL, Ebraheim NA. Anatomy of the palmar cutaneous branch of the median nerve: a review. J Orthop 2019; 16:5769.

21. van den Broeke LR, Theuvenet WJ, van Wingerden JJ. Effectiveness of mini-open carpal tunnel release: an outcome study. Arch Plast Surg 2019;46:350-8.

22. Castillo R, Sheth K, Babigian A, et al. Recurrent carpal tunnel syndrome associated with extension of flexor digitorum muscle bellies into the carpal tunnel: a case series. Arch Plast Surg 2018;45:474-8. 